Measuring Agents' Reaction to Private and Public Information in Games with Strategic Complementarities

Camille Cornand, Franck Heinemann

December 2013 


\section{GATE Groupe d'Analyse et de Théorie Économique Lyon-St Étienne}

93, chemin des Mouilles 69130 Ecully - France

Tel. +33(0)4 72866060

Fax $+33(0) 472866090$

6, rue Basse des Rives 42023 Saint-Etienne cedex 02 - France

Tel. +33 (0)4 77421960

Fax. +33 (0)4 77421950

Messagerie électronique / Email : gate@gate.cnrs.fr

Téléchargement / Download : http://www.gate.cnrs.fr - Publications / Working Papers 


\title{
Measuring Agents' Reaction to Private and Public Information in Games with Strategic Complementarities*
}

\author{
Camille Cornand \\ Université de Lyon, Lyon, F-69007, France ; \\ CNRS, GATE Lyon Saint-Etienne, Ecully, \\ F-69130, France
}

Email: cornand@gate.cnrs.fr

\author{
Frank Heinemann \\ Technische Universität Berlin \\ Chair of Macroeconomics, H 52 \\ Straße des 17. Juni 135 \\ 10623 Berlin, Germany \\ Email: frank.heinemann@ww.tu-berlin.de
}

February 19, 2013

\begin{abstract}
In games with strategic complementarities, public information about the state of the world has a larger impact on equilibrium actions than private information of the same precision, because public signals are more informative about the likely behavior of others. We present an experiment in which agents' optimal actions are a weighted average of the fundamental state and their expectations of other agents' actions. We measure the responses to public and private signals. We find that, on average, subjects put a larger weight on the public signal. In line with theoretical predictions, as the relative weight of the coordination component in a player's utility increases, players put more weight on the public signal when making their choices. However, the weight is smaller than in equilibrium, which indicates that subjects underestimate the information contained in public signals about other players' beliefs.
\end{abstract}

JEL Classification: C92, D82, D84.

Keywords: coordination games, strategic uncertainty, private information, public information.

\section{1 - Introduction}

Financial markets, macroeconomic environments, and network games are often characterized by strategic complementarities. In games with strategic complementarities, public and private information about an uncertain state of the world have distinct effects, because information that is shared by several agents is more informative about the likely beliefs and actions of others than private information. Since agents have an incentive to align their actions to the expected actions of others, equilibrium choices are affected more by public than by private signals of equal precision. This has been used to explain why markets 'overreact' to public signals, so that public information may raise price volatility on financial markets and cause negative welfare effects. The aim of this paper is to experimentally evaluate the weights that subjects attach to private and public signals in games of weak

\footnotetext{
* We are thankful to Gabriel Desgranges, John Duffy, Petra Geraats, Ed Hopkins, Paul Pezanis-Christou, and Alexander Meyer-Gohde for helpful comments, to Romain Zeiliger for programming Regate, to Kene Boun My for providing assistance, to the students for their participation in the experiment and to the Conseil Scientifique de l'Université de Strasbourg and the ANR-DFG joint grant for financial support.
} 
strategic complementarities ${ }^{1}$. We show that on average agents indeed attach a higher weight to public than to private information, but the asymmetry in weights is smaller than in equilibrium.

Our experiment builds upon Morris and Shin (2002), who present a generalized beauty contest game with weak strategic complementarities for analyzing the distinct effects of public and private information. In their game, an agent's action is a weighted average of a public and a private signal. The equilibrium weight attached to the public signal is higher than its relative precision. This 'overreaction' (Morris and Shin (2002), p. 1530) is due to the higher informational content of public signals regarding the likely beliefs of other agents. In this context, Morris and Shin show that raising the precision of public signals may reduce expected welfare. ${ }^{2}$ Welfare effects of public and private information depend on two considerations requiring empirical investigation: the relative precision of the two signals and the relative weight that agents attach to these signals. These considerations interact: the higher the weight that agents attach to public signals, the more likely it is that an imprecise public signal will reduce welfare. In equilibrium, the relative weight is determined by the signals' relative precision and agents' payoff functions.

This paper precisely aims at measuring and analyzing how much public signals are taken into account compared to private ones - in a game with weak strategic complementarities. We present an experiment on a game that is characterized by both fundamental and strategic uncertainty. As in Morris and Shin (2002), agents have to choose actions that are close to a fundamental state but also close to each other. We test predictions of this approach by implementing two-player versions of this game with varying weights on fundamental and strategic uncertainty. In line with theory, we find that: (i) subjects put larger weights on the public signal and (ii) as the relative weight of the coordination component in a player's utility rises, players put more weight on the public signal. However, overall, the weights put on the public signal are smaller than theoretically predicted.

A cognitive hierarchy model with 2 levels of reasoning helps explaining the observation that subjects attach a lower weight on public signals than predicted by the equilibrium. Stated second order beliefs, however, indicate that subjects underestimate how informative the public signal is in assessing other players' expectations. This may be viewed as an alternative explanation of behavior. However, these non-Bayesian higher-order beliefs can explain only a part of the observed systematic deviation of behavior from equilibrium.

The next section presents the basic game. Section 3 sets the experimental design and motivates hypotheses. Section 4 states the experimental results and provides measures for the relative

\footnotetext{
1 Angeletos and Pavan (2004) distinguish weak and strong strategic complementarities. Under weak complementarities, the game has a unique equilibrium, while strong strategic complementarities define a coordination game with multiple equilibria.

${ }^{2}$ Svensson (2006) has argued that detrimental welfare effects of public information are unlikely, because they require that public information be of lower precision than private information, which conflicts with empirical findings. For a response, see Morris et al. (2006). For discussions about the welfare effects of public information in closely related frameworks, see Cornand and Heinemann (2008), Baeriswyl and Cornand (2011), Woodford (2005), Angeletos and Pavan (2007 a,b), Hellwig (2005), and Myatt and Wallace (2008) among others.
} 
weights that subjects put on private and common signals, relating them to equilibrium predictions. Section 5 presents two behavioral theories to explain the observed deviation of behavior from equilibrium. Finally, section 6 concludes.

\section{2 - Two-player game with weak strategic complements}

We describe a game in which agents have to choose actions that are close to a fundamental state but also close to what the others believe. The framework is a two-player version of Morris and Shin (2002) adapted for conducting an experiment. ${ }^{3}$

The fundamental state of nature is given by $\theta$ and has a uniform distribution on the reals. Agent $i$ chooses action $a_{i} \in \mathfrak{R}$, and we write $a$ for the action profile over all agents. The utility function for individual $i$ has two loss components:

$$
u_{i}(a, \theta) \equiv U_{0}-(1-r)\left(a_{i}-\theta\right)^{2}-r\left(a_{i}-a_{j}\right)^{2},
$$

where $j$ is the partner of $i$ and $U_{0}$ is a constant that cannot be affected by player $i$. The first quadratic term captures losses arising from the distance between an action and the underlying state $\theta$. The second quadratic term describes losses from miscoordination between both players' actions. Finally, $r \in[0,1]$ indicates the relative weight attributed to the coordination component. The coordination component is reminiscent of Keynes' beauty contest.

Agents face uncertainty concerning $\theta$. They receive two kinds of signals that deviate from $\theta$ by some error terms with uniform distribution. All agents receive a public (common) signal $y \sim U[\theta-\varepsilon, \theta+\varepsilon]$. In addition, each agent receives a private signal $x_{i} \sim U[\theta-\varepsilon, \theta+\varepsilon]$. Noise terms $x_{i}-\theta$ of distinct individuals and the noise of the public signal $y-\theta$ are independent and their distribution is treated as exogenously given.

The optimal action of agent $i$ is given by the first order condition from maximizing (1):

$$
a_{i}=(1-r) E_{i}(\theta)+r E_{i}\left(a_{j}\right),
$$

where $E_{i}(\cdot)$ is the posterior expectation conditional on $x_{i}$ and $y$ and $a_{j}$ is the action of the other player $j$. One needs to calculate $E_{i}(\theta)$ and $E_{i}\left(a_{j}\right)$. Because both signals have the same precision,

$$
E_{i}(\theta)=E\left(\theta \mid x_{i}, y\right)=\frac{x_{i}+y}{2}=E\left(x_{j} \mid x_{i}, y\right) \text {. }
$$

The optimal strategy of any agent is a linear combination of his signals: $a_{j}=\gamma x_{j}+(1-\gamma) y$. So,

\footnotetext{
${ }^{3}$ Compared to the model of Morris and Shin (2002), we required the number of players to be finite and we also changed the distributions of the signals from normal to uniform in order to have a simple distribution with bounded support for the experiment. In Morris and Shin, the constant $U_{0}$ adds the average losses from miscoordination, so that the coordination part becomes a zero-sum game and aggregate welfare depends only on the distance between actions and fundamental state.
} 


$$
E_{i}\left(a_{j}\right)=E\left(a_{j} \mid x_{i}, y\right)=\gamma E\left(x_{j} \mid x_{i}, y\right)+(1-\gamma) y=(1-\gamma) y+\gamma \frac{x_{i}+y}{2} .
$$

Inserting this in equation (2) yields

$$
a_{i}=r E_{i}\left(a_{i} \mid x_{i}, y\right)+(1-r) E_{i}\left(\theta \mid x_{i}, y\right)=\frac{x_{i}[(1-r(1-\gamma))]+y[1+r(1-\gamma)]}{2} \text {. }
$$

Thus, in equilibrium $\gamma^{*}=\frac{1-r\left(1-\gamma^{*}\right)}{2}$, which is equivalent to $\gamma^{*}=\frac{1-r}{2-r}$. Agent $i$ 's equilibrium action is now given by $a_{i}^{*}=\frac{y+(1-r) x_{i}}{2-r}$. The equilibrium weight on private information $\gamma^{*}$ falls short of its weight of $1 / 2$ in the Bayesian expectation of the fundamental, because the public signal conveys more information about the likely action of the other player than the private signal.

In equilibrium, actions are distorted away from the expected $\theta$ towards $y$. This mirrors the disproportionate impact of the public signal in coordinating agents' actions and emphasizes the role of public information as a focal point for private actions. Note that the equilibrium weight on the private signal $\gamma^{*}$ decreases in the relative importance of coordination measured by $r$. Morris and Shin (2002) concentrate on the welfare effects of public information and show that if welfare depends only on how close actions are to the fundamental, public signals of low precision may actually reduce ex-ante expected welfare, even though they improve agents' ability to predict the fundamental. Here, the negative welfare effect from distorting actions towards the public signal exceeds the positive welfare effect that arises from the additional information. In these environments, the economy is better off without a public signal. This argument has been used to question the merits of central bank transparency.

Negative welfare effects of public signals require that agents put a sufficiently large weight on public signals. In beauty contest games like the one used by Morris and Shin (2002), real players' behavior has been found consistent with limited levels of reasoning (Nagel, 1995). As will be explained in Section 5 below, such behavior leads to weights on the public signal that are lower than in equilibrium. Cornand and Heinemann (2012) show that negative welfare effects of public disclosures require that agents put weights on public signals that are at least as large as the weight associated with level-2 reasoning. Thus, it is an empirical question, whether real players put a weight on public signals that is sufficiently strong to cause negative welfare effects of public signals. ${ }^{4}$ To

\footnotetext{
${ }^{4}$ Other experiments concerning the welfare effects of public information have concentrated on games with multiple equilibria: Anctil et al. (2004) demonstrate that private signals with high precision are not sufficient to achieve coordination on an efficient equilibrium. Heinemann et al. (2004) compare perfect public and noisy private signals in a speculative attack game and find small effects towards higher efficiency with perfect information. Cornand (2006) shows that subjects put a larger weight on the public signal if they receive both a private and a public signal about the state of the economy. For other experiments dealing with public versus private information, see Forsythe et al. (1982), Plott and Sunder (1988), McKelvey and Ordeshook (1985), McKelvey and Page (1990) and Hanson (1996). They investigate how individuals use public information to
} 
answer this question, we propose an experiment that measures the actual weights that subjects put on private versus public information.

\section{3 - Experimental design}

The experiment aims at measuring reactions to private and public information in the game introduced in the last section. In particular, we are interested in testing whether subjects put larger weights on public than on private signals, whether the observed weights are in line with equilibrium predictions, and how changes in $r$ affect these weights.

18 sessions were run at the BETA (Bureau d'Economie Théorique et Appliquée) laboratory in Strasbourg (using software Regate (Zeiliger, 2000)) between January 2008 and March 2010. Each session had 16 participants who were mainly students from Strasbourg University (most were students in economics, mathematics, biology and psychology). Subjects were seated in random order at PCs. Instructions were then read aloud and questions answered in private. Throughout the sessions, students were not allowed to communicate with one another and could not see each others' screens. Each subject could only participate in one session. Before starting the experiment, subjects were required to answer a few questions to ascertain their understanding of the rules. Instructions and questionnaires are given in Appendix A. The experiment started after all subjects had given the correct answers to these questions.

Each session consisted of four stages with a total of 50 periods. While Stages 1, 2, and 4 were the same in all sessions, Stage 3 contained three different treatments across sessions. In each period, subjects were paired up randomly. They did not know the identity of their partner and they knew that they would most likely not meet the same partner in the next period. For each pair of subjects, a fundamental state $\theta$ was drawn randomly using a uniform distribution from the interval [50, 450]. Each subject received a private signal $x_{i} \in[\theta-10, \theta+10]$. In addition, each pair of subjects received a common (public) signal $y \in[\theta-10, \theta+10]$. Signals were drawn independently from these intervals using a uniform distribution. ${ }^{5}$ The random process was explained in the instructions.

In the first three stages, each subject had to decide on an action $a_{i}$ conditional on her signals. ${ }^{6}$ Signals and actions were limited to one decimal point.

\footnotetext{
augment their original private information and whether, in doing so, a rational expectations equilibrium is attained.

${ }^{5}$ Having a sufficiently large support of the prior distribution enables us to reduce the informational content conveyed by the prior mean. In addition, this reduces the set of signals, for which the conditional posterior distribution is skewed.

${ }^{6}$ To limit potential losses that might arise from typing mistakes, we restricted choices and stated expectations to the interval $[y-20, y+20]$. This restriction was not mentioned in the instructions. If a subject entered a number outside this interval, the screen displayed a message indicating that her choice is too far away from signals. Apparently, this restriction was not binding. We observed only one (out of 12,960) decision situations, where a subject actually chose an action at the border of this interval, and less than 50 decisions, where the difference between action and public signal exceeded 15 . Some subjects actually reported typing mistakes.
} 
In the first stage ("r=0"), the payoff function was given by $100-\left(a_{i}-\theta\right)^{2}$. Here, subjects should choose an action as close as possible to the fundamental state, independent from their partners' choices. This stage aimed at familiarizing subjects with the game form and controlling for their Bayesian rationality. Since both signals have the same precision, the rational choice is $a_{i}=\left(x_{i}+y\right) / 2$ whenever both signals are in the interval $[60,440]^{7}$

In the second stage (" $r=1$ "), the payoff function was given by $100-\left(a_{i}-a_{j}\right)^{2}$, where $a_{i}$ denotes the partner's action. Here, subjects should coordinate their actions irrespective of the fundamental state: this is a pure coordination game from which subjects should learn that public information is more helpful in overcoming coordination problems than private information. Any strategy that maps the public signal into the reals is an equilibrium ${ }^{8}$, provided that both subjects coordinate on the same strategy. The public signal, however, provides a focal point, ${ }^{9}$ so that we expected subjects to coordinate on actions $a_{i}=y$.

Stage 3 contained three different treatments, each conducted in 6 of the sessions. In Treatment " $r=0.5$ ", the payoff function was $200-\left(a_{i}-a_{j}\right)^{2}-\left(a_{i}-\theta\right)^{2}$, while it was $400-\left(a_{i}-a_{j}\right)^{2}-3\left(a_{i}-\theta\right)^{2}$ in Treatment " $r=0.25$ " and $400-3\left(a_{i}-a_{j}\right)^{2}-\left(a_{i}-\theta\right)^{2}$ in Treatment " $r=0.75$ ". Contrary to Stages 1 and 2, the three treatments of Stage 3 exhibit both a fundamental and a coordination motive. They correspond to the game by Morris and Shin (2002). Given that players choose linear combinations of both signals $a_{j}=\gamma x_{j}+(1-\gamma) y$, the equilibrium weight ${ }^{10}$ on the private signal in Stage 3 is $\gamma^{*}=(1-r) /(2-r)$. Note that the limit of $\gamma^{*}$ for $r \rightarrow 1$ selects the equilibrium with $a_{i}=y$ for $r=1$.

In Stage 4 we elicited higher-order beliefs. Subjects also received private and public signals. But here, each subject was asked to state two expectations: one regarding the fundamental state and one regarding her partner's expectation of the fundamental state. The payoff function was

\footnotetext{
${ }^{7}$ When signals are smaller than 60 or larger than 440 , the posterior distribution of $\theta$ is skewed, because of the bounded support of $\theta$.

${ }^{8}$ In the limiting case where fundamental uncertainty disappears and subjects' payoffs depend only on the distance between their actions, equilibrium theory does not yield a unique prediction. Any coordinated strategy is an equilibrium. However, the limit of equilibria in games with a decreasing weight on fundamental uncertainty uniquely selects a strategy in which all agents follow the public signal and ignore all private information.

${ }^{9} \mathrm{~A}$ formalization of focal points is provided by Alós-Ferrer and Kuzmics (2008).

${ }^{10}$ Due to the bounded support of $\theta$, the equilibrium deviates from this linear combination into the direction of the center of the support. To see this, imagine that player $i$ receives signals $x_{i}=40$ and $y=50$. From her private signal, she can deduce that $\theta=50$. The posterior distribution of the other player's signal is uniform in $[40,60]$. Since the other player should never choose an action below 50, the expected action by the other player is above 50. Therefore, player $i$ should also choose an action above 50. Obviously, it is too demanding to assume that players update their beliefs correctly at the edges of the support. In the data, we find systematic effects for signals up to 60 and above 440 . We checked that restricting data analysis to situations with $70<\theta<430$ does not alter our results.
} 
100- $\left(e_{i}(\theta)-\theta\right)^{2}-\left(e_{i}\left(e_{j}(\theta)\right)-e_{j}(\theta)\right)^{2}$, where $e_{i}(\cdot)$ denotes the stated expectation of subject $i$. The last term of the payoff function incentivizes the statement of higher-order beliefs. To our knowledge, this is the first experiment with a direct elicitation of higher-order beliefs.

Since individual decisions are not independent in repeated coordination games, we conducted 18 sessions with at least 6 sessions per treatment. ${ }^{11}$ For statistical tests, we use the average weight on the private signal within a session as an independent observation. Table 1 gives an overview over sessions, stages, and treatments.

\begin{tabular}{|c|c|c|c|c|}
\hline Sessions & Stage 1 & Stage 2 & Stage 3 & Stage 4 \\
\hline 1 to 6 & \multirow{3}{*}{$\begin{array}{c}\text { " } r=0 ” \\
(5 \text { periods) }\end{array}$} & \multirow{3}{*}{$\begin{array}{c}\text { " } r=1 ” \\
\text { (10 periods) }\end{array}$} & $\begin{array}{c}\text { Treatment } \\
\text { "r=0.5” } \\
\text { (30 periods) }\end{array}$ & \multirow{3}{*}{$\begin{array}{l}\text { Belief Elicitation } \\
\quad(5 \text { periods })\end{array}$} \\
\hline 7 to 12 & & & $\begin{array}{l}\text { Treatment } \\
\text { " } r=0.25 " \\
(30 \text { periods) }\end{array}$ & \\
\hline 13 to 18 & & & $\begin{array}{c}\text { Treatment } \\
\text { " } r=0.75 " \\
(30 \text { periods })^{12}\end{array}$ & \\
\hline
\end{tabular}

Table 1 - Composition of the different sessions

After each period, subjects were informed about the true state, their partner's decision and their payoff. Information about past periods from the same stage (including signals and own decisions) was displayed during the decision phase on the lower part of the screen. Subjects knew from the start that they were playing the aforementioned stages in this order. At the end of each session, the points earned were summed up and converted into Euros. 100 points were converted to 25 cents in sessions 1 -6 and $13-18$, and to 43 cents in sessions $7-12$. Payoffs ranged from $€ 12$ to $€ 33$. The average payoff was about $€ 22 .{ }^{13}$ Sessions lasted for around 90 minutes.

Our main hypotheses are derived from the theory predictions: Table 2 summarizes the equilibrium weights on the private signal $\gamma^{*}$ for interior states. The natural hypothesis arising from theory is that subjects attach weights to private signals that are distributed around the equilibrium weights. As explained above, we intend to test this hypothesis against the alternative arising from a cognitive hierarchy model which predicts higher weights on the private signal for $r>0$. Two other testable hypotheses are consistent with equilibrium and with limited levels of reasoning: the weights put on the private signal are smaller than 0.5 (for $r>0$ ) and decreasing in $r$.

\footnotetext{
${ }^{11}$ We also conducted 6 control sessions to include treatments with only one signal in order to test the welfare reducing effect of private signals for $r=1$ directly by comparing payoffs. Control sessions elicited beliefs at the second stage before strategic games and with a slightly different representation of the payoff function. We only present standard sessions in the main text. The results of control sessions are reported in Appendix B.

${ }^{12}$ In Session 13, Treatment " $r=0.75$ " unintentionally stopped after 26 periods.

${ }^{13}$ In all stages, it was possible to earn negative points. This actually occurred in about $3.3 \%$ of all decision situations in standard sessions. Realized losses were of a size that could be counterbalanced by positive payoffs within a few periods. In general, losses were covered by earnings from the next three periods or balanced by earnings of previous periods.
} 


\begin{tabular}{l|lllll}
\hline$r$ & 0 & 0.25 & 0.5 & 0.75 & $\rightarrow 1$ \\
\hline$\gamma^{*}$ & 0.500 & 0.429 & 0.333 & 0.200 & $\rightarrow 0$ \\
\hline
\end{tabular}

Table 2 - Equilibrium weight $\gamma^{*}$ as a function of $r$

\section{4 - Experimental results}

We use the following structure in analyzing data. First, we analyze whether subjects' choices are between their two signals as predicted by theory. Here we also look at the proportion of choices that are closer to the public or private signal contingent on the stage or treatment. Then, we estimate the average weights that subjects attach to the private signal for each session, stage, and treatment. We investigate whether average weights on the private signal are positive, smaller than 0.5 , and whether they deviate systematically from the equilibrium value. If subjects best respond to the observed actions of their respective partners, their strategies should converge over time towards the equilibrium. We test whether there is a time trend by comparing the data from the first and second halves of a stage. Comparing observed weights from different treatments, we test comparative static predictions arising from theory.

\subsection{Some considerations about rationality}

Theory predicts that subjects choose actions that are weighted averages of their two signals. Hence, actions should be contained in $I_{1}=\left[\min \left(y, x_{i}\right), \max \left(y, x_{i}\right)\right]$. However, we observe that about $10 \%$ of all decisions are outside this interval, in particular if both signals are very close to each other. To get a better impression, how far these choices go outside, we also look whether choices are consistent with the contingent support of the state's distribution. From her signals, subject $i$ can deduce that the true state of the world is contained in the interval $I_{2}=\left[\max \left(y, x_{i}\right)-10, \min \left(y, x_{i}\right)+10\right]$. We observe that the proportion of choices outside this interval also becomes large when the interval is small. Furthermore, the smaller is $I_{1}$, the larger is $I_{2}$. Since both of these intervals have some appeal for reasonable choices, we check, how many choices were contained in the union of both sets, $I_{3}=I_{1} \cup I_{2}$. Note that the size of interval $I_{3}$ is less sensitive to the randomly drawn signals and never gets smaller than 10 , while the size of $I_{1}$ and $I_{2}$ may shrink to zero for appropriate signals.

Result 1: $98 \%$ of choices are contained in $I_{3}$. The proportion of choices inside $I_{1}$ is $85 \%$. 


\begin{tabular}{|c|c|c|c|c|c|}
\hline Value of $r$ & 0 & 0.25 & 0.5 & 0.75 & 1 \\
\hline Inside $I_{1}=\left[\min \left(y, x_{i}\right), \max \left(y, x_{i}\right)\right]$ & $75 \%$ & $85 \%$ & $86 \%$ & $86 \%$ & $86 \%$ \\
\hline Choice $=y$ & $1 \%$ & $1 \%$ & $7 \%$ & $21 \%$ & $33 \%$ \\
\hline Closer to $y$ & $29 \%$ & $34 \%$ & $44 \%$ & $35 \%$ & $26 \%$ \\
\hline Middle $(+/-0.05)$ & $14 \%$ & $18 \%$ & $11 \%$ & $10 \%$ & $9 \%$ \\
\hline Closer to $x_{i}$ & $31 \%$ & $31 \%$ & $24 \%$ & $20 \%$ & $18 \%$ \\
\hline Choice $=x_{i}$ & $1 \%$ & $1 \%$ & $1 \%$ & $0.4 \%$ & $1 \%$ \\
\hline Outside $I_{1}$ beyond $y$ & $12 \%$ & $8 \%$ & $8 \%$ & $8 \%$ & $8 \%$ \\
\hline Outside $I_{1}$ beyond $x_{i}$ & $12 \%$ & $8 \%$ & $6 \%$ & $7 \%$ & $6 \%$ \\
\hline Inside $I_{2}=[\max (Y, X)-10 ; \min (y, X)+10]$ & $94 \%$ & $97 \%$ & $96 \%$ & $91 \%$ & $88 \%$ \\
\hline Inside $I_{3}=I_{1} \cup I_{2}$ & $97 \%$ & $99 \%$ & $99 \%$ & $99 \%$ & $98 \%$ \\
\hline
\end{tabular}

Table 3 - Crude classification of individual choices

Table 3 displays the percentage of all choices within these intervals. Counting the number of choices that are closer to the common signal, closer to the private, or in the middle provides a crude first impression of whether subjects put a larger weight on the common signal and how dispersed the distribution of relative weights is. ${ }^{14}$ As $r$ is increased, the proportion of choices closer or equal to the common signal $y$ increases.

\subsection{Estimated weights on the private signal}

We estimate the relative weights of the two signals on subjects' decisions by fitting linear regressions to data. Because the payoff function rewards coordination when $r>0$, we may expect that the weights applied by different subjects within a session converge over time. Hence, individual weights are not independent. To be on the conservative side, our main statistical analyses are based on the average weights per session. We estimate these weights by joining the data from all subjects within a session. The regression equation is

$$
a_{i t}-y_{t}=c+\gamma\left(x_{i t}-y_{t}\right)+u_{i t},
$$

where $\gamma$ is the session-specific relative weight on private information. The constant $c$ stands for a bias towards higher or lower numbers. Using the Bonferroni correction for multiple hypotheses, this constant turns out to be insignificant in all stages and treatments. Implicitly, our regression imposes a restriction that the absolute coefficients on private and common signals add up to 1 . In regressions without this restriction, we checked that violations of this restriction are not significant either. ${ }^{15}$

Table 4 displays average weights on the private signal for all sessions and stages and compares them with theoretical weights. For games that were played at least 10 rounds, we split the data from the first and second halves of the stage so that we could analyze whether there was some systematic change of behavior over time. Figure 1 presents the evolution of these weights over periods; this representation captures the differences across stages within each group of sessions and between

\footnotetext{
${ }^{14}$ To break ties in counting: if both signals and the action coincide, we count the choice as "Middle". If $\left|y-x_{i}\right|=0.1$, and $a_{i}=x_{i}\left[a_{i}=y\right]$, we count the choice as "equal to $x_{i}$ " ["equal to $y$ "].

${ }^{15}$ Data and programs are available at http://www.macroeconomics.tu-berlin.de/menue/working_papers/.
} 
different treatments across sessions. Note that average weights on private signals stay above the equilibrium values (for $r>0$ ) in all periods.

\begin{tabular}{cccccccccc}
\hline Session & $r=0$ & $\begin{array}{c}r=1, \\
\text { 1st half }\end{array}$ & $\begin{array}{c}r=1, \\
\text { 2nd half }\end{array}$ & $\begin{array}{c}r=0.5, \\
\text { 1st half }\end{array}$ & $\begin{array}{c}r=0.5, \\
\text { 2nd half }\end{array}$ & $\begin{array}{c}r=0.25, \\
\text { 1st half }\end{array}$ & $\begin{array}{c}r=0.25, \\
\text { 2nd half }\end{array}$ & $\begin{array}{c}r=0.75, \\
1 \text { st half }\end{array}$ & $\begin{array}{c}r=0.75, \\
\text { 2nd half }\end{array}$ \\
\hline 1 & 0.522 & 0.266 & 0.158 & 0.419 & 0.408 & & & & \\
2 & 0.506 & 0.369 & 0.219 & 0.452 & 0.463 & & & & \\
3 & 0.570 & 0.318 & 0.390 & 0.453 & 0.447 & & & & \\
4 & 0.475 & 0.380 & 0.344 & 0.416 & 0.475 & & & & \\
5 & 0.453 & 0.328 & 0.098 & 0.340 & 0.393 & & & & \\
6 & 0.516 & 0.349 & 0.200 & 0.409 & 0.453 & & & & \\
7 & 0.435 & 0.353 & 0.185 & & & 0.485 & 0.492 & & \\
8 & 0.472 & 0.346 & 0.209 & & & 0.498 & 0.466 & & \\
9 & 0.579 & 0.398 & 0.363 & & & 0.521 & 0.500 & & \\
10 & 0.496 & 0.339 & 0.135 & & & 0.480 & 0.460 & & \\
11 & 0.559 & 0.286 & 0.195 & & & 0.454 & 0.475 & & \\
12 & 0.494 & 0.257 & 0.210 & & & 0.477 & 0.473 & & \\
13 & 0.497 & 0.376 & 0.205 & & & & & 0.417 & 0.391 \\
14 & 0.504 & 0.190 & 0.208 & & & & & 0.309 & 0.294 \\
15 & 0.492 & 0.468 & 0.387 & & & & & 0.405 & 0.457 \\
16 & 0.489 & 0.390 & 0.350 & & & & & 0.408 & 0.377 \\
17 & 0.501 & 0.352 & 0.162 & & & & & 0.289 & 0.187 \\
18 & 0.555 & 0.389 & 0.331 & & & & 0.338 \\
\hline Average & 0.506 & 0.342 & 0.242 & 0.415 & 0.440 & 0.486 & 0.478 & 0.353 & 0.341 \\
St.dev. & 0.039 & 0.063 & 0.092 & 0.041 & 0.032 & 0.022 & 0.015 & 0.063 & 0.093 \\
\hline Equilibrium & 0.5 & 0 & 0 & 0.333 & 0.333 & 0.429 & 0.429 & 0.2 & 0.2 \\
weight & & & & & & & & & \\
\hline
\end{tabular}

Table 4 - Group specific weights on the private signal

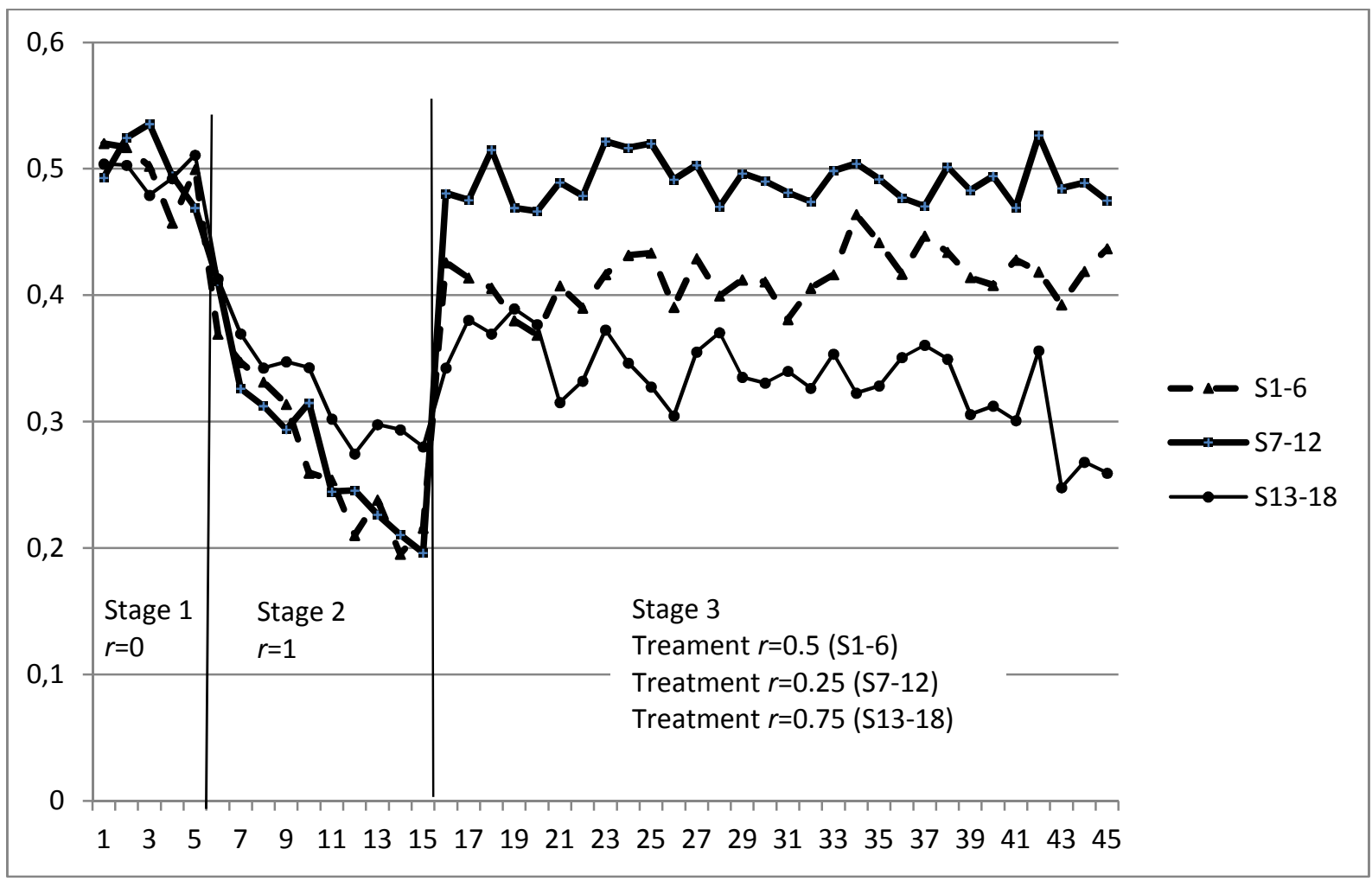

Figure 1 - Evolution of average weights on the private signal

Data indicate that the relative weight on the private signal is around 0.5 for " $r=0$ " and tends to be decreasing in $r$. For $r=1, r=0.5$, and $r=0.25$, all session-specific weights are larger than in equilibrium. 
For $r=0.75$, there is one exception (Session 17, $2^{\text {nd }}$ half). Comparing first and second halves, there seems to be some trend towards equilibrium in stage " $r=1$ ", but not in treatments with $0<r<1$.

These impressions are widely confirmed by nonparametric tests, reported below. Specifically, we test whether estimated weights differ from 0.5 , from the respective equilibrium values, and between first and second halves of a stage/treatment. We also test monotony of the weights in $r$. Unless otherwise noted, all tests are based on counting session-specific averages as independent observations with a significance level of $5 \%$. When we split data from the first and second halves of a stage, we apply tests to both halves separately.

Result 2: For $r=0$, subjects put an equal weight on both signals consistent with Bayesian rationality.

In Stage " $r=0 "$, there is no significant difference between group specific estimated weights and 0.5. Two-tailed Wilcoxon matched pairs signed rank tests yield p-values of 0.77 . Since individual decisions are independent in this first stage, we also performed tests on estimated individual weights. ${ }^{16}$ The hypothesis that individual weights are distributed around 0.5 cannot be rejected either (the p-value is 0.70$)$.

Our main result is that for $r>0$, subjects put a lower weight on public signals than in equilibrium.

Result 3: For $0<r<1$, subjects tend to put larger weights on public than on private signals, but the difference is smaller than theoretically predicted. There is no trend towards equilibrium.

Our prior expectation (arising from the widespread success of cognitive hierarchy models in beauty contest games) was that estimated group-specific weights on the private signal are smaller than 0.5 and larger than equilibrium weights. Therefore, we use one-sided tests on the null hypotheses that the median weight is equal to the respective equilibrium value against the alternative hypotheses that it is smaller than 0.5 or exceeds the equilibrium weight, respectively. One-tailed Wilcoxon matched pairs signed rank tests yield the p-values, reported in Table 5. While most tests reject the null hypotheses at $5 \%$, we cannot reject that the median weight equals 0.5 for the first half of Treatment " $r=0.25$ ".

\begin{tabular}{lllllllll}
\hline $\begin{array}{l}\text { Null } \\
\text { hypothesis }\end{array}$ & $\begin{array}{l}\text { Alternative } \\
\text { hypothesis }\end{array}$ & $\begin{array}{l}r=0.5, \\
1 \text { st half }\end{array}$ & $\begin{array}{l}\text { 2nd half } \\
\text { hyst half }\end{array}$ & $\begin{array}{l}\text { 2nd half } \\
\text { 1st half }\end{array}$ & 2nd half \\
\hline$\gamma=0.5$ & $\gamma<0.5$ & 0.015 & 0.015 & 0.106 & 0.030 & 0.015 & 0.015 \\
$\gamma=0.429$ & $\gamma>0.429$ & & & 0.015 & 0.015 & & \\
$\gamma=0.333$ & $\gamma>0.333$ & 0.015 & 0.015 & & & & \\
$\gamma=0.2$ & $\gamma>0.2$ & & & & & 0.015 & 0.031 \\
\hline
\end{tabular}

Table 5 - P-values of one-tailed Wilcoxon matched pairs signed rank tests.

\footnotetext{
${ }^{16}$ Average weights per individual are estimated by a similar procedure as average weights per session, with a separate regression for each subject.
} 
Comparing the weights assigned to private signals in the first and second halves of each treatment, we find no significant differences. Using the two-tailed Wilcoxon matched pairs test, pvalues range between $15 \%$ and $85 \%$. In particular, for Treatment " $r=0.75$ ", the p-value is $85 \%$. Thus, we conclude that subjects tend to put larger weights on public than on private information ${ }^{17}$, but the difference is smaller than theoretically predicted and there is no systematic trend over time.

Result 4: For $r=1$, subjects assign larger weights to public than to private information: they lean towards coordinating on the public signal, but do not achieve full coordination during the course of the stage.

In Stage " $r=1$ ", all estimated weights are positive and below 0.5. Hence, the Wilcoxon matched pairs signed rank test yields p-values below $1 \%$ for the null hypotheses $\gamma=0$ and $\gamma=0.5$. Testing whether the weights assigned to private signals in the last 5 rounds were equal to those in the first half against the alternative of a systematic trend towards equilibrium (i.e. lower weights in the last rounds), the onetailed Wilcoxon matched pairs test yields p-values of $0.02 \%$. Thus, we can reject the hypothesis of no trend towards equilibrium for this stage.

Result 5: Over all sessions, the weight assigned to the private signal tends to decrease in $r$ as predicted by theory.

A one-tailed Wilcoxon matched pairs signed rank test finds that $\gamma$ is getting significantly smaller with increasing $r$, if we compare " $r=0$ " with " $r=0.5$ " or " $r=0.5$ " with " $r=1$ ". P-values are always below $4 \%$. Note that here the difference in $r$ is 0.5. Comparing treatments where the difference in $r$ is only 0.25 , test results are mixed: comparing " $r=0.5$ " with " $r=0.25$ " or " $r=0.5$ " with " $r=0.75$ ", one-tailed MannWhitney tests yields p-values below $3 \%$ except for " $r=0.5$ " (first half) compared to " $r=0.75$ " (second half), where $\mathrm{p}=6.6 \%$. However, the one-tailed Wilcoxon matched pairs fails to reject the hypothesis of equal weights for Treatment " $r=0.25$ " versus " $r=0$ " and for Treatment " $r=0.75$ " versus " $r=1$ " (p>9\%) except for Treatment " $r=0.75$ " (second half) compared to " $r=1$ " (second half), where $p=3.1 \%$. Figure 2 relates the average weight on the private signal for each treatment to theoretical predictions and also displays how the weight depends on $r$.

\footnotetext{
${ }^{17}$ This result seems to be corroborated by subjects' written comments in the post-experimental questionnaire (this questionnaire is presented in Appendix A.3). 43\% of subjects explicitly wrote (without being directly asked) that $y$ is more informative than $x_{i}$ on the other participant's decision. The percentage of subjects reporting such statements is positively correlated to the value of $r$ in stage 3 .
} 


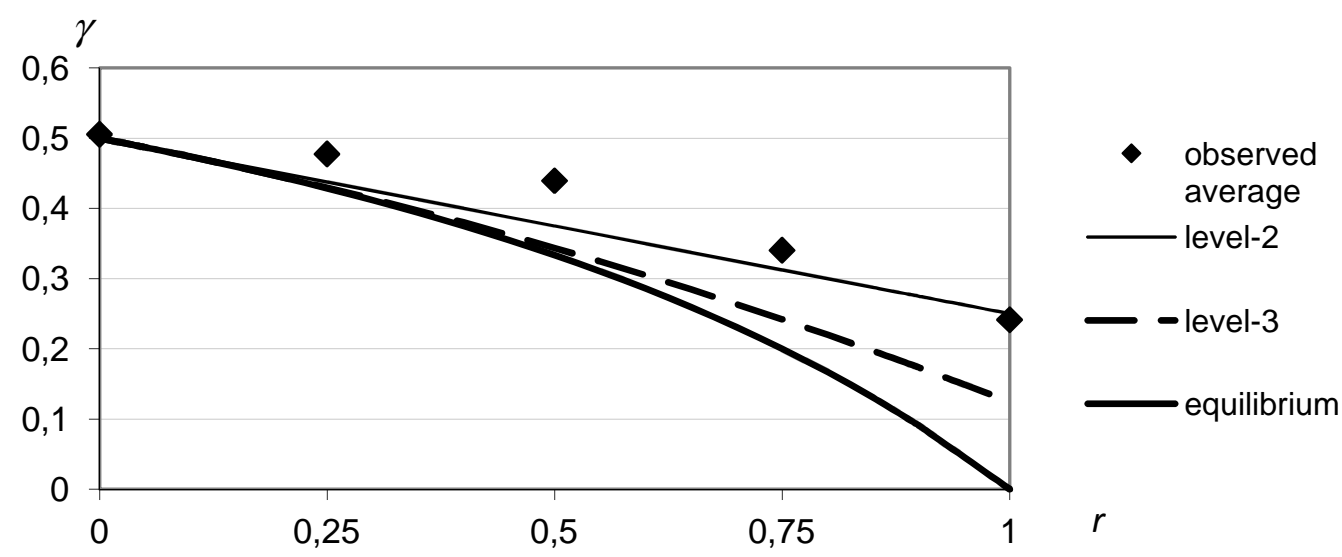

Figure 2 - Weight on the private signal for different values of $r$

\section{Alternative explanations for observed behavior}

We know from Nagel (1995), Kübler and Weizsäcker (2004) and many other experimental papers that the equilibrium may be a poor predictor for actual behavior when it requires infinite levels of reasoning. In their environments, cognitive-hierarchy models with limited levels of reasoning yield better predictions. ${ }^{18}$ In this section, we study two models of bounded rationality: one is a model of a cognitive hierarchy (limited levels of reasoning) and the other one is based on non-Bayesian higherorder beliefs. Both models are able to explain weights on the private signal that exceed the equilibrium values. ${ }^{19}$

\subsection{Cognitive hierarchy}

The model of cognitive hierarchies assumes that agents process information rationally, but fail to consider that others decide with the same degree of rationality. Nagel (1995) and Stahl and Wilson (1994) define level-0 types as subjects who choose an action randomly from a uniform distribution over all possible actions. For $k>0$, a level- $k$ type is playing best response to level- $k-1$. In our game, if the partner's action has a uniform distribution on the reals, expected losses from the coordination part of the payoff function are infinite for any action, so that any action is a best reply as long as $r>0$. However, expected losses resulting from deviations from the fundamental are minimized by choosing the expected state. Hence, we define the best response to a uniform distribution over all reals as

\footnotetext{
${ }^{18}$ Shapiro, Shi, and Zillante (2009) analyze the predictive power of level- $k$ reasoning in a game that combines features of Morris and Shin (2002) with Nagel (1995). They try to identify whether individual strategies are consistent with level- $k$ reasoning. They argue that the predictive power of level- $k$ reasoning is positively related to the strength of the coordination motive and to the symmetry of information. Their experiment however treats public and private signals in an asymmetric way by normalizing public signals.

${ }^{19}$ Hypotheses that arise from combining non-Bayesian higher-order beliefs with limited levels of reasoning are derived and tested in Appendix D.
} 
$a_{i}^{1}=E_{i}(\theta)$, so that level-1 players place weight 0.5 on both signals. Note that $a_{i}^{1}=E_{i}(\theta)$ is the best response of a player who ignores the strategic part of the payoff function. Starting from this definition, actions for higher levels of reasoning can be calculated as follows: suppose that a player $j$ attaches weight $\gamma_{k}$ to her private signal. The best response to such behavior is

$$
a_{i}^{k+1}=(1-r) E_{i}(\theta)+r E_{i}\left(a_{j}\right)=(1-r) E_{i}(\theta)+r \gamma_{k} E_{i}\left(x_{j}\right)+r\left(1-\gamma_{k}\right) y .
$$

Since the expected private signal of the other player equals the expected state,

$$
a_{i}^{k+1}=\left[(1-r)+r \gamma_{k}\right] E_{i}(\theta)+r\left(1-\gamma_{k}\right) y=\frac{(1-r)+r \gamma_{k}}{2} x_{i}+\left[\frac{(1-r)+r \gamma_{k}}{2}+r\left(1-\gamma_{k}\right)\right] y .
$$

Hence, the next level of reasoning is $\gamma_{k+1}=\frac{(1-r)+r \gamma_{k}}{2}$. With higher levels, the weight on the private signal decreases from 0.5 towards the equilibrium value.

Table 6 summarizes the resulting weights on the private signal for increasing levels of reasoning and the equilibrium weight on the private signal $\gamma^{*}$ for different values of $r$. Comparing observed weights with these theoretical values allows testing the cognitive hierarchy model.

\begin{tabular}{lllllll}
\hline Value of $r$ & Level-1 & $\begin{array}{c}\text { Level-2 } \\
\gamma_{2}\end{array}$ & $\begin{array}{l}\text { Level-3 } \\
\gamma_{3}\end{array}$ & $\begin{array}{l}\text { Level-4 } \\
\gamma_{4}\end{array}$ & $\ldots$ & $\begin{array}{l}\text { Equilibrium weight } \\
\text { (infinite levels of reasoning) } \gamma^{*}\end{array}$ \\
\hline 0 & & 0.50 & 0.50 & 0.50 & $\ldots$ & 0.50 \\
0.25 & 0.50 & 0.437 & 0.429 & 0.429 & $\ldots$ & 0.429 \\
0.5 & 0.50 & 0.375 & 0.344 & 0.336 & $\ldots$ & 0.333 \\
0.75 & 0.50 & 0.312 & 0.242 & 0.216 & $\ldots$ & 0.2 \\
1 & 0.50 & 0.25 & 0.125 & 0.062 & $\ldots$ & 0 \\
\hline
\end{tabular}

Table 6 - Theoretical weight on $x_{i}$ depending on $r$ and on the level of reasoning

Result 6: For interior values of $r$, most estimated group weights on private signals are close to or above the weights given by level-2 reasoning.

As can be seen from Figure 2, the observed average weights are close to the weights of level-2 reasoning for all values of $r$. Comparing session-specific weights presented in Table 4 with weights from limited levels of reasoning displayed in Table 6, shows that in Treatments " $r=0.5$ " and " $r=0.25$ ", average group weights are higher than the weights from level-2 reasoning with only one exception for $r=0.5$ (Session 5, first half). The hypothesis that average weights are equal to or below those from level-2 reasoning is rejected by one-tailed Wilcoxon matched pairs signed rank test at p-values below $5 \%$. In Treatment " $r=0.75$ ", session-specific weights are distributed around the weight from level-2, and we cannot reject that the weights are equal to or below those from level-2 reasoning. In this treatment, one-sided tests reject level-3 reasoning (observed weights are not lower than $\gamma_{3}$ ), but twosided tests do not (equality of observed weights with $\gamma_{3}$ cannot be rejected). 
For $r=1$, second half, the hypothesis $\gamma \leq \gamma_{2}$ cannot be rejected either. These inhomogeneous test results indicate that subjects might behave in accordance to higher levels of reasoning with increasing $r$.

As Cornand and Heinemann (2012) show, the welfare-detrimental effects of public information require that agents put a weight on the private signal that is smaller than the one associated with level-2 reasoning. Since we cannot reject this for $r \geq 0.75$, we cannot rule out that for a strong coordination motive (high value of $r$ ), public information may be detrimental to welfare, if welfare depends only on how close actions are to the fundamental as in Morris and Shin (2002).

\subsection{Non-Bayesian higher-order beliefs}

Weights on the private signal above equilibrium values can also be explained by systematic mistakes in the formation of higher-order beliefs. For using information rationally, a subject has to form conditional expectations about his partner's beliefs based on his own information. Theoretically, subjects should put a weight of 0.5 on the private signal when estimating $\theta$ and a weight of 0.25 in estimating the other's estimation of $\theta$. This does not require any assumptions about others' behavior apart from Bayesian rationality. If subject $j$ has rational first-order beliefs, then $E_{j}(\theta)=0.5 x_{j}+0.5 y$ and rational second-order beliefs are $E_{i}\left(E_{j}(\theta)\right)=0.5 E_{i}(\theta)+0.5 y=0.25 x_{i}+0.75 y$. More generally, if $E_{j}(\theta)=\lambda x_{j}+(1-\lambda) y$, then $E_{i}\left(E_{j}(\theta)\right)=\lambda E_{i}(\theta)+(1-\lambda) y=0.5 \lambda x_{i}+(1-\lambda / 2) y$.

Previous studies have explored whether subjects update beliefs according to Bayes' rule, when information arrives sequentially. Various studies from the 1960s found that "[u]pon receipt of new information, subjects revise their posterior probability estimates in the same direction as the optimal model [Bayes' rule], but the revision is typically too small" (Slovic and Lichtenstein, 1971, 693). Recent studies explore whether subjects are Bayesian in updating self assessments. While some find significant deviations from Bayes' rule (Eil and Rao, 2011), others report that subjects update overconfident prior beliefs correctly (Grossman and Owens, 2010). To our knowledge there is no previous experimental study exploring whether subjects use Bayes' rule in forming higher-order beliefs. Dale and Morgan (2012) test some predictions of Morris and Shin (2002) comparing treatments with private and public signals of different precisions. The weights that subjects put on signals of low precision and on the prior (which also has a low precision for indicating the fundamental) are much higher than under Bayesian belief formation. Since in their experiment public signals always have a low precision, they find higher weights on the public signal than we do. They find weights that are distributed around the equilibrium values and negative welfare effects of public signals. ${ }^{20}$ In our experiment, all signals have the same precision, so that we can detect mistakes in the

\footnotetext{
${ }^{20}$ In the light of our previous impression that the level of reasoning may be correlated with $r$, the high parameter of $r=0.8$, chosen by Dale and Morgan (2012), may contribute to higher weights on public signals. They also inform subjects about the best response after each round which may enforce convergence to equilibrium.
} 
formation of higher-order beliefs without this potentially confounding bias towards low quality signals.

Result 7: Subjects attach too high a weight to the private signal when predicting other subjects' beliefs about fundamentals.

Data from belief elicitation reveal that subjects attach (on average) a weight of 0.463 on the private signal when estimating $\theta$ and an average weight of 0.291 when estimating their partner's estimation of $\theta$. Two-tailed Wilcoxon tests on estimated group-specific weights reject the hypotheses that the median weight in estimating $\theta$ equals $0.5(\mathrm{p}=0.005)$ in favor of lower weights on private signals, and reject that the median weight in estimating the other player's estimation equals 0.25 $(\mathrm{p}=0.038)$ in favor of higher weights on private signals. 229 out of 288 subjects attribute a weight higher than 0.25 to the private signal in this task. Although Bayesian rationality requires that the weight on the private signal in second-order beliefs be half of the weight in estimating $\theta$, it is in fact $62.2 \%$ and thereby too high to be consistent with Bayesian rationality. This can explain a part of the differences between observed weights and equilibrium predictions in the other stages. As we elicited beliefs directly after Stage $3(0<r<1)$, we may expect that higher-order beliefs in the later periods of Stage 3 are formed in about the same way as elicited beliefs. However, as we show in Appendix C, the deviations from Bayesian rationality in forming higher-order beliefs are too small to explain the whole difference between observations and equilibrium predictions in the second half of Stage 3.

It is surprising, though, that subjects underused private signals in forming their expectation of $\theta$, especially in light of the results from Stage 1 (" $r=0$ ") that is equivalent to asking first-order expectations. We attribute this to an order effect: after 40 periods in Stages 2 and 3, in which the public signal was more important than the private, some subjects may have developed a routine in putting higher weights on public signals that was applied to both tasks in Stage 4. In Appendix C, we present the results of 6 control sessions, in which we elicited beliefs directly after Stage 1 and before all stages, in which common signals are theoretically more important than private ones. Here, we find that the weights on the private signal in first-order expectations are distributed around 0.5 (equality cannot be rejected) while the weights in second-order expectations are all above 0.3 and on average $83 \%$ of the weight in first-order expectations. Here, the violation of Bayesian rationality in forming higher-order beliefs is even more pronounced. These results indicate that subjects underestimate how informative public signals are for estimating other players' beliefs.

\section{6 - Conclusion}

Our experiment provides convincing data for the hypothesis that subjects attach larger weights to public than to private signals if they have incentives to coordinate their actions. The experiment also investigates whether players put higher weights on the public signal if the relative importance of the 
coordination component is increased. We find clear evidence in line with this qualitative prediction of equilibrium theory. The observed weights are, however, lower than equilibrium values. They are closer to the weights arising from level-2 reasoning of a cognitive hierarchy model, for which Cornand and Heinemann (2012) show that public information cannot be welfare detrimental.

\section{References}

Alós-Ferrer, C., and C. Kuzmics (2008), "Hidden Symmetries and Focal Points,“ University of Konstanz, TWI Research Paper no. 35.

Anctil, R., J. Dickhaut, C. Kanodia, and B. Shapiro (2004), "Information Transparency and Coordination Failure: Theory and Experiment," Journal of Accounting Research, 42, 159-195.

Angeletos, G.-M., and A. Pavan (2004), "Transparency of Information and Coordination in Economies with Investment Complementarities," American Economic Review (Papers and Proceedings), 94, 91-98.

Angeletos, G.-M., and A. Pavan (2007a), "Socially optimal coordination: Characterization and policy implications," Journal of the European Economic Association (Papers and Proceedings), 5, 585593.

Angeletos, G.-M., and A. Pavan (2007b), "Efficient Use of Information and Social Value of Information," Econometrica, 75, 1103-1142.

Baeriswyl, R., and C. Cornand (2011), "Reducing overreaction to central banks' disclosures : theory and experiment," Working Paper du GATE Lyon Saint-Etienne, 41.

Cornand, C. (2006), "Speculative Attack and Informational Structure: An Experimental Study," Review of International Economics, 14, 797-817

Cornand, C., and F. Heinemann (2008), "Optimal Degree of Public Information Dissemination," The Economic Journal, 118, 718-742.

Cornand, C., and F. Heinemann (2012), "Higher-Order Beliefs and the Social Value of Public Information," mimeo.

Dale, Donald J., and John Morgan (2012), "Experiments on the Social Value of Public Information," mimeo.

Eil, D., and J.M. Rao (2011), "The Good News-Bad News Effect: Asymmetric Processing of Objective Information about Yourself," American Economic Journal: Microeconomics, 3, 114138.

Forsythe, R., T.R. Palfrey, and C.R. Plott (1982), "Asset Valuation in an Experimental Market," Econometrica, 50, 537-567.

Grossman, Z., and D. Owens (2010), “An Unlucky Feeling: Persistent Overestimation of Absolute Performance with Noisy Feedback," University of California at Santa Barbara, Economics Working Paper No. 1937484.

Hanson, R. (1996), "Correction to MacKelvey and Page, "Public and Private Information: An Experimental Study of Information Pooling"," Econometrica, 64, 1223-1224.

Heinemann, F., R. Nagel, and P. Ockenfels (2004), "The Theory of Global Games on Test: Experimental Analysis of Coordination Games with Public and Private Information," Econometrica, 72, 1583-1599.

Hellwig, C. (2005), "Heterogeneous Information and the Welfare Effects of Public Information Disclosures," UCLA, mimeo.

Kübler, D., and G. Weizsäcker (2004), "Limited Depth of Reasoning and Failure of Cascade Formation in the Laboratory," Review of Economic Studies, 71, 425-442. 
Lorenzoni, G. (2010), "Optimal Monetary Policy with Uncertain Fundamentals and Dispersed Information," Review of Economic Studies, 77, 305-338.

McKelvey, R. D., and P. C. Ordeshook (1985), "Elections with Limited Information: A Fulfilled Expectations Model Using Contemporaneous Poll and Endorsement Data as Information Sources," Journal of Economic Theory, 35, 55-85.

McKelvey, R. D., and T. Page (1990), "Public and Private Information: An Experimental Study of Information Pooling," Econometrica, 58, 1321-1339.

Morris, S., and H.S. Shin (2002), "Social Value of Public Information," American Economic Review, 92, 1522-1534.

Morris, S., and H.S. Shin (2004), "Coordination Risk and the Price of Debt," European Economic Review, 48, 133-153.

Morris, S., H. S. Shin, and H. Tong (2006), "Social Value of Public Information: Morris and Shin (2002) Is Actually Pro-Transparency, Not Con: Reply," American Economic Review, 96, 453-55.

Myatt, D.P., and C. Wallace (2008), "On the sources and value of information: public announcements and macroeconomic performance," University of Oxford, Dept. of economics discussion paper no. 411.

Nagel, R. (1995), "Unraveling in Guessing Games: An Experimental Study," American Economic Review, 85, 1313-1326.

Plott, C.R., and S. Sunder (1988), "Rational Expectations and the Aggregation of Diverse Information in Laboratory Security Markets," Econometrica, 56, 1085-1118.

Roca, M. (2010), "Transparency and Monetary Policy with Imperfect Common Knowledge," IMF Working Paper 10/91.

Romer, C., and D. Romer (2000), "Federal Reserve Private Information and the Behavior of Interest Rates," American Economic Review, 90, 429-457.

Shapiro, D., X. Shi, and A. Zillante (2009), "Robustness of Level- $k$ Reasoning in Modified Beauty Contest Games," mimeo.

Slovic, P., and S. Lichtenstein (1971), "Comparison of Bayesian and Regression Approaches to the Study of Information Processing in Judgment," Organizational Behavior and Human Performance, 6, 649-744.

Stahl, D. O., and P.W. Wilson (1994), "Experimental Evidence on Players' Models of Other Players," Journal of Economic Behavior and Organization, 25, 309-327.

Svensson, L.E.O. (2006), "Social Value of Public Information: Comment: Morris and Shin (2002) Is Actually Pro-Transparency, Not Con,” American Economic Review, 96, 448-452.

Woodford, M. (2005), "Central Bank Communication and Policy Effectiveness," in: The Greenspan Era: Lessons for the Future, Kansas City: Federal Reserve Bank of Kansas City, 399-474.

Zeiliger, R. (2000), "A Presentation of Regate, Internet based Software for Experimental Economics," http://www.gate.cnrs.fr/ zeiliger/regate/RegateIntro.ppt, GATE. 